\title{
COVID-19: Epidemiology and Preventive Measures in Jiangsu Province, China
}

\author{
Chen $Y^{1}$, Liu J ${ }^{2}$, Yang $C^{1}$, Zhang $A^{3 *}$ and $A i Z^{3 *}$ \\ ${ }^{1}$ Tongji University School of Medicine, Shanghai, China \\ ${ }^{2}$ Center for Data Science, Peking University, Beijing, \\ China \\ ${ }^{3}$ Department of Medical Statistics, School of Medicine, \\ Tongji University, Shanghai, China \\ *Corresponding author: Aihong Zhang, Department \\ of Medical Statistics, School of Medicine, Tongji \\ University, 1239 Siping Road, Shanghai-200092, China
}

Received: J uly 05, 2021; Accepted: August 04, 2021; Published: August 11, 2021

\begin{abstract}
Objective: To analyze the epidemiological features of coronavirus disease 2019 (COVID-19) in Jiangsu Province after starting level 1 response to major public health emergencies and assess the effectiveness of epidemic prevention and control measures took by the government.
\end{abstract}

Methods: Individual information on all novel coronavirus pneumonia confirmed cases from January 10, 2020 to March $18^{\text {th }}$ in Jiangsu was collected from the official website of Health Commission of Jiangsu province and its cities, Hubei Health Commission and the Chinese Health Commission. The trends in demographic and epidemiological characteristics of confirmed cases were estimated and the real time reproduction number $\left(R_{t}\right)$ were calculated.

Results: 631 COVID-19 cases were diagnosed in Jiangsu Province, covering 13 districts in Jiangsu. The confirmed cases consisted of imported cases (30.27\%) and local transmitted cases (69.73\%). 64.15\% (238/371) local transmitted cases had reported a history of exposure to COVID-19 cases or patients with acute respiratory infection. Close contact with COVID-19 infectors in families, parties or some public places is the main transmission route for local transmission cases. The epidemic peak occurred from January 30 to February 4 , then began to decline. The ratio of male to female cases was 1.22 to 1 , and most patients were in the group of $30-70$ years old, 49 patients $(7.8 \%)$ with mild symptom and 572 patients (90.6\%) with common type accounted for the majority. As of March 18, the cities with higher cumulative confirmed cases were Nanjing (93 cases, 15\%), Suzhou (87 cases, 14\%) and Xuzhou (79 cases, $12 \%)$. The $\mathrm{R}_{\mathrm{t}}$ reached a peak of $4.05(95 \% \mathrm{Cl} 2.27-6.34)$ on January 20 , then began to decline. $R_{t}$ was below the epidemic threshold $\left(R_{t}<1\right)$ after January 31 and maintained a continuing downward trend.

Conclusion: The epidemic preventive and control measures have contributed to interrupt local transmission of COVID-19 in Jiangsu Province, and the epidemic in Jiangsu Province was well under control in less than a month.

Keywords: COVID-19; Epidemiology; Prevention; Reproduction number

\section{Abbreviations}

SARS-CoV-2: Severe Acute Respiratory Syndrome Coronavirus 2; COVID-19: Coronavirus Disease 2019; WHO: World Health Organization

\section{Background}

Since December 2019, a cluster of cases of pneumonia of unknown cause was detected in Wuhan City, Hubei Province, China. A novel coronavirus had been identified in samples obtained from cases and that initial analysis of virus genetic sequences suggested that this was the cause of the outbreak. This virus is referred to as SARS-CoV-2, and the associated disease as COVID-19 (Coronavirus Disease 2019). SARS-CoV-2 belongs to the coronavirus of the genus $\beta$, with an envelope, with round or oval virus particles, polymorphic, and the diameter is $60-40 \mathrm{~nm}$. The continued human-to-human transmission of SARS-CoV-2 is reported, which can be spread through respiratory droplets and close contact, resulting in a rapid increase of confirmed cases nationwide and even worldwide [1-3]. On January 30, the epidemic was declared by WHO as 'a Public Health Emergency of
International Concern'. As of 10 April 2020, more than 1.56 million cases have been diagnosed globally, with over 95,000 fatalities. Investigating the epidemiological characteristics of COVID-19 is essential for formulating and implementing effective prevention and control strategies and measures. There are few studies on the epidemiology features and transmission dynamics of novel coronary pneumonia in Jiangsu Province. This study aimed to describe the epidemiological characteristics of COVID-19 cases occurring in Jiangsu province until March 18, 2020, and to estimate the real time reproduction numbers to assess whether the prevention and control measures have been effective in blocking transmission.

\section{Methods}

\section{The data source}

The data source is based on the official data of Jiangsu Commission of Health (http://wjw.jiangsu.gov.cn), from which the confirmed cases, including epidemics information and baseline information, were counted from January 23 to March 18, 2020. Information on Gender, age, history of sojourn in Hubei, clinical classification,
Austin J Orthopade \& Rheumatol - Volume 8 Issue 2 - 202 ISSN: 2472-369X | www.austinpublishing group.com Chen et al. () All rights are reserved
Citation: Chen Y, Liu J, Yang C, Zhang A and Ai Z. COVID-19: Epidemiology and Preventive Measures in Jiangsu Province, China. Austin J Orthopade \& Rheumatol. 2021; 8(2): 1101. 
epidemiologic linkage to other COVID-19 cases or areas were extracted from the government websites [4,5]. The diagnosis of patients with COVID-19, and the clinical classification of mild, common, severe and critical diseases, the discharged standard and the isolation standard were all judged according to [Novel Coronary Virus Pneumonia Diagnosis and Treatment Program] (Trial Sixth Edition) [6-8]. All the respiratory secretions were confirmed to be positive for novel coronavirus nucleic acid by laboratory test. At the same time, a series of prevention and control policies and measures took by the government of Jiangsu Province were also collected from the government websites. We also obtained the data from the websites of Hubei Health Commission and the Chinese Health Commission.

\section{Definition of COVID-19's clinical classification}

- $\quad$ Mild patient: Patients present mild clinical symptoms and no pneumonia imaging characterization;

- Common type: Patient had fever, respiratory tract and other symptoms, with pneumonia imaging manifestations;

- Severe and critically ill patients: Patients had any of the following symptoms: 1) Respiratory distress, RR $>30$ beats/min; 2) The oxygen saturation is less than $93 \%$ under the resting state; 3 ) Partial pressure of oxygen in arterial blood ( $\mathrm{Pa} 02)$ / oxygen concentration (Fi02) < 30mHg (1mmHg=0.133kpa); 4) Shock; 5) Combined with other organ function exhaustion and ICU monitoring treatment is needed.

\section{Definition of imported cases and local transmission cases}

- Imported cases: Nucleic acid test positive with a travelling history of Hubei or residence in overseas epidemic countries or regions within 14 days before the onset.

- Local transmission case: Nucleic acid test positive without an epidemic area travelling history within 14 days.

\section{Statistical analysis}

Using histogram and line chart to describe the time trends of confirmed cases, cured cases and severe cases respectively. The geographic locations of confirmed cases were plotted according to three key time points. The mean of the time dependent (or real time) reproduction number $\left(\mathrm{R}_{\mathrm{t}}\right)$ was estimated over 5 and 7 days moving by a Bayesian approach respectively, with the mean and standard deviation of serial interval being 7.5 and 3.4 respectively, considering importations and local transmission $[2,10,11]$. Statistical analysis was conducted with R4.0, GraphPad Prism6.0 and ArcGis10.6.

\section{Result}

\section{Characteristics of COVID-19 cases}

Until March 18, 2020, a total of 631 confirmed cases were reported, including 191 imported cases (30.27\%), 440 local transmitted cases (69.73\%). 64.15\% (238/371) local transmitted cases had reported a history of exposure to COVID-19 cases or patients with acute respiratory infection (Table 1).

Since the demographic sociological information of cases was not a project that must be announced by the Commission of Health, the basic information of some cases was missing. The distribution of 559 (88.59\%) diagnosed patients was analyzed. Of the 559 confirmed cases, 307 were male $(54.92 \%)$ and 252 were female (45.08\%) (Figure 1).
Table 1: Characteristics of COVID-19 cases in Jiangsu Province.

\begin{tabular}{|c|c|c|c|}
\hline \multirow{2}{*}{ Characteristics } & \multicolumn{3}{|c|}{ Confirmed cases (n (\%)) } \\
\hline & Combined & Imported & Local transmitted \\
\hline Total & $631(100)$ & $191(30.27)$ & $440(69.73)$ \\
\hline \multicolumn{4}{|l|}{ Age group \# (year) } \\
\hline $0 \sim 9$ & $6(1.07)$ & $3(1.63)$ & $3(0.80)$ \\
\hline $10 \sim 19$ & $13(2.33)$ & $3(1.63)$ & $10(2.67)$ \\
\hline $20 \sim 29$ & $68(12.16)$ & $19(10.33)$ & $49(13.07)$ \\
\hline $30 \sim 39$ & $130(23.26)$ & $49(26.63)$ & $81(21.60)$ \\
\hline $40 \sim 49$ & $107(19.14)$ & $42(22.83)$ & 65 (17.33) \\
\hline $50 \sim 59$ & $127(22.72)$ & $43(23.37)$ & $84(22.40)$ \\
\hline $60 \sim 69$ & $71(12.70)$ & $22(11.96)$ & 49 (13.07) \\
\hline $70 \sim 79$ & $28(5.01)$ & $5(2.72)$ & $23(6.13)$ \\
\hline$\geq 80$ & $9(1.61)$ & $0(0.00)$ & $9(2.40)$ \\
\hline \multicolumn{4}{|l|}{ Gender group ${ }^{\#}$} \\
\hline male & $307(54.92)$ & $115(62.50)$ & $192(51.20)$ \\
\hline female & $252(45.08)$ & $69(37.50)$ & $183(48.80)$ \\
\hline \multicolumn{4}{|l|}{ Time } \\
\hline Before 28-Jan & 70 (11.09) & $68(35.60)$ & $2(0.45)$ \\
\hline From 29-Jan to 30-Jan & $59(9.35)$ & $46(24.08)$ & $13(2.95)$ \\
\hline From 31-Jan to 7-Feb & $279(44.22)$ & $77(40.32)$ & $202(45.92)$ \\
\hline From 8-Feb to 19-Feb & $223(35.34)$ & $0(0.00)$ & $223(50.68)$ \\
\hline
\end{tabular}

\#: Only 559 (88.59\%) of total 631 cases were reported by age and gender.

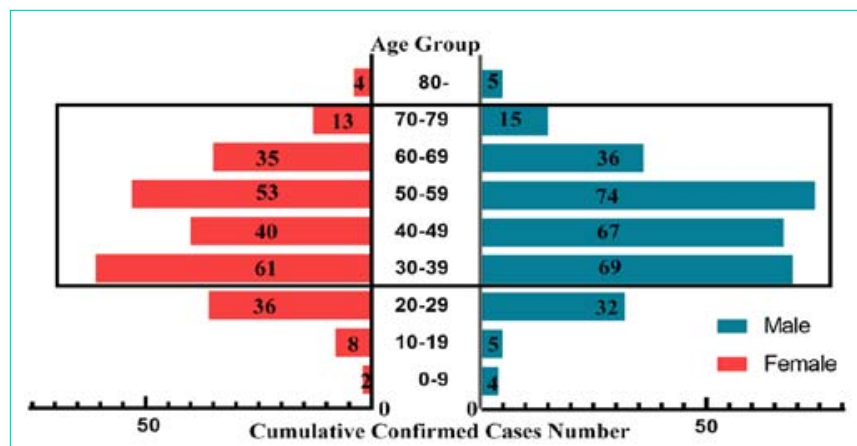

Figure 1: Age and gender distribution of confirmed patients in Jiangsu Province.

The male to female gander ratio was 1.22: 1 , which meant more male infected than female. And among 559 cases, 19 cases were younger than the 20-year-old (3.40\%), 68 cases were among $20-30$ years old (12.16\%), and 108 cases were old than the 80 -year-old (1.61\%). The number of confirmed cases in 30-80 years old is the largest, 463 cases, accounting for $82.83 \%$ of the total number, which was roughly similar to the overall situation in China (30-80 years old group accounted for $86.6 \%)$ [5]. The average age of diagnosed cases was 46.15 ( \pm 15.52$)$ years for males and $44.91( \pm 16.99)$ years for females. The youngest patient was a 10-month-old male child in Nanjing, and the oldest patient was a 91-year-old male patient in Huaian city.

\section{Geographical distribution}

On January 22, 2020, 5 newly diagnosed COVID-19 cases were first confirmed by Jiangsu Commission of Health. All 5 patients 


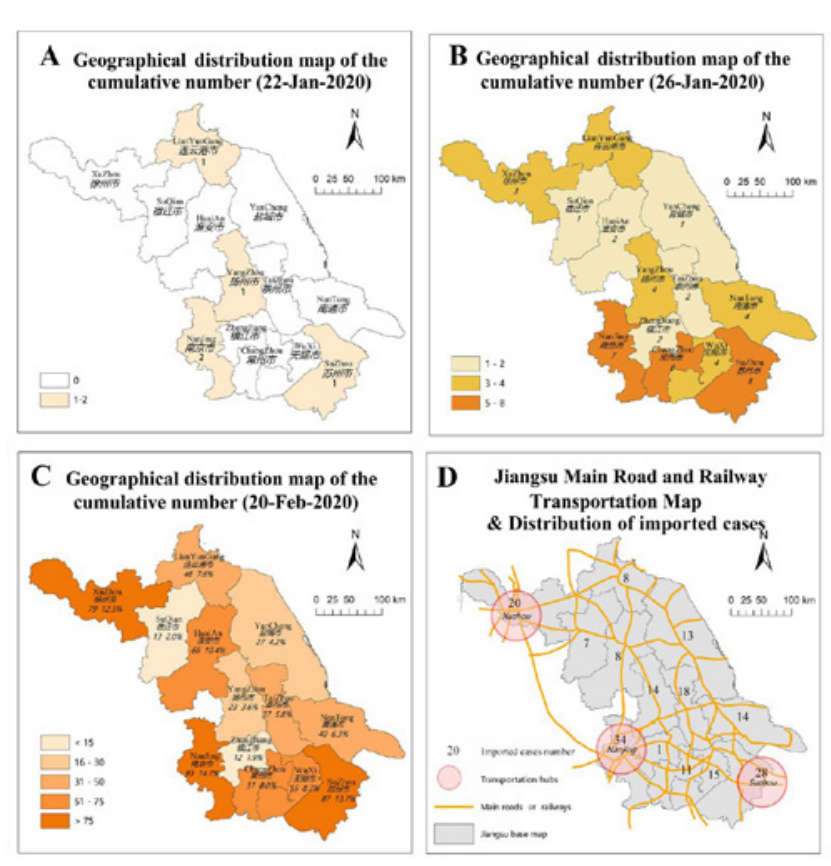

Figure 2: Geographical distribution of the cumulative number of COVID-19 patients in Jiangsu.

A: Geographical distribution map of Jiangsu on 22-Jun.

B: Geographical distribution map of Jiangsu on 26-Jun.

C: Geographical distribution map of Jiangsu on 20-Feb, D: Jiangsu main road and railway transportation map.

had a Hubei travelling history which means imported cases. Two of them were confirmed by Nanjing City, and one each was in Suzhou, Yangzhou, and Lianyungang (Figure 2A). Four days later, an imported case who was also the $47^{\text {th }}$ confirmed case in Jiangsu province was diagnosed in Zhenjiang City for the first time. All 13 cities across the province had reported confirmed COVID-19 cases up to Jan 26 [4]. Nanjing, Changzhou and Suzhou have more COVID-19 patients, compared with other cities (Figure 2B). Until March 18, Jiangsu Province had a total of 631 cases of COVID-19 diagnosed, in which the top three cities of the confirmed cases number were Nanjing (93 cases, 15\%), Suzhou (87 cases, 14\%), Xuzhou (79 cases, 12\%) (Figure 2C). Interestingly, out of a total of 191 imported cases until February 3 , these three cities are also the top three in terms of the imported cases number (Figure 2D).

\section{Time distribution}

The epidemic curve of confirmed cases showed three stages: upward, plateau and downward. In the initial stage new confirmed cases everyday were on the rise, reached the peak from January 30 to February 6, then began to decline. On January 27, Jiangsu Commission of Health confirmed the first case of second-generation transmission of novel coronary pneumonia in the province. It means imported cases and local transmission cases coexist in Jiangsu province. The new additions number after February 8 showed a downward trend in volatility. The confirmed cases number stopped increasing on February 19, with total 631 cases. Through a retrospective study of patients' epidemiologic history, imported cases were predominantly diagnosed before January 29 , the percentage of daily imported cases accounted for more than $67 \%$ in daily total cases. Then the imported

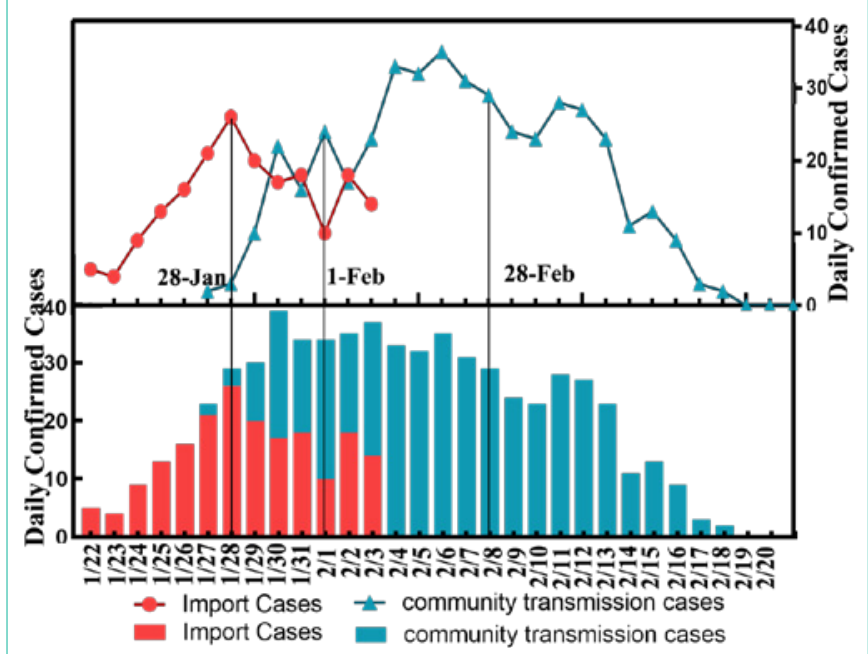

Figure 3: The number and source of newly diagnosed cases in Jiangsu Province.

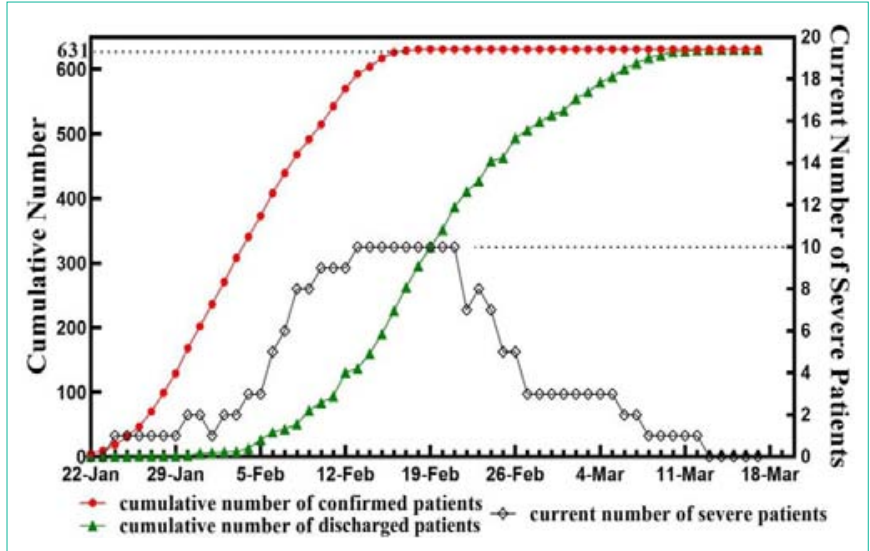

Figure 4: Line chart of cumulative confirmed patient number, Cumulative discharged patient number, current severe patient number in Jiangsu Province.

cases number began to decline and no imported cases were reported after February 3. From that time on the confirmed cases are all secondgeneration or above transmission cases in the province, which means, the onset was typically caused by SARS-CoV-2 after contacting with confirmed cases in the gathering place (Figure 3). Meanwhile, we analyzed median, lower and upper quartiles time from symptom onset to diagnosis in 292 patients with complete information, with a median time from symptom onset to diagnosis of 7 (5-10) days.

From February 18 to March 18, there were no newly confirmed cases in Jiangsu Province. On the basis of the aggregation data from the website of Jiangsu Commission of Health, there were a total of 631 confirmed COVID-19 patients in Jiangsu province, within which 49 mild patients (7.8\%), 572 common patients (90.6\%), and 10 severe and critically ill patients (1.6\%) (Figure 4). As to March 18, all 631 confirmed cases in Jiangsu Province were discharged from hospital, according to the discharge standard reference that body temperature returned to normal for more than 3 days, respiratory symptoms improved significantly, and respiratory pathogenic nucleic acid test was negative for two consecutive times (sampling interval at least 1 day) [6-8]. 


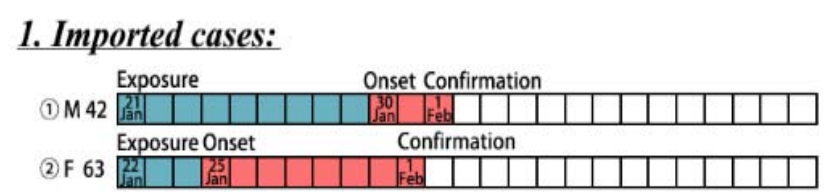

\section{Second-generation transmission cases:}

\subsection{Family aggregation}

Contact with his father

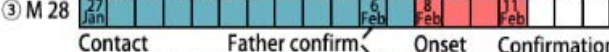

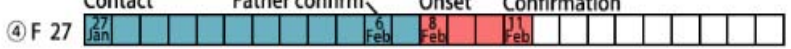

2.2 Entertainment aggregation

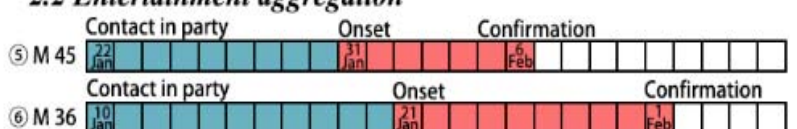

\subsection{Unconscious close contact}

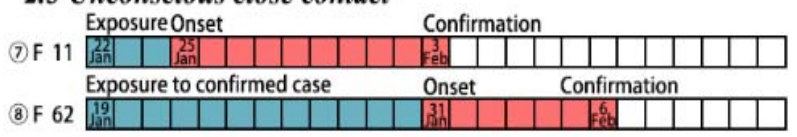

(1) Gender Age $\square$ One day $\square$ exposure to onset $\square=\square_{\text {onset to confirmation }}^{\text {Time from }}$

Figure 5: Examples of transmission routes.

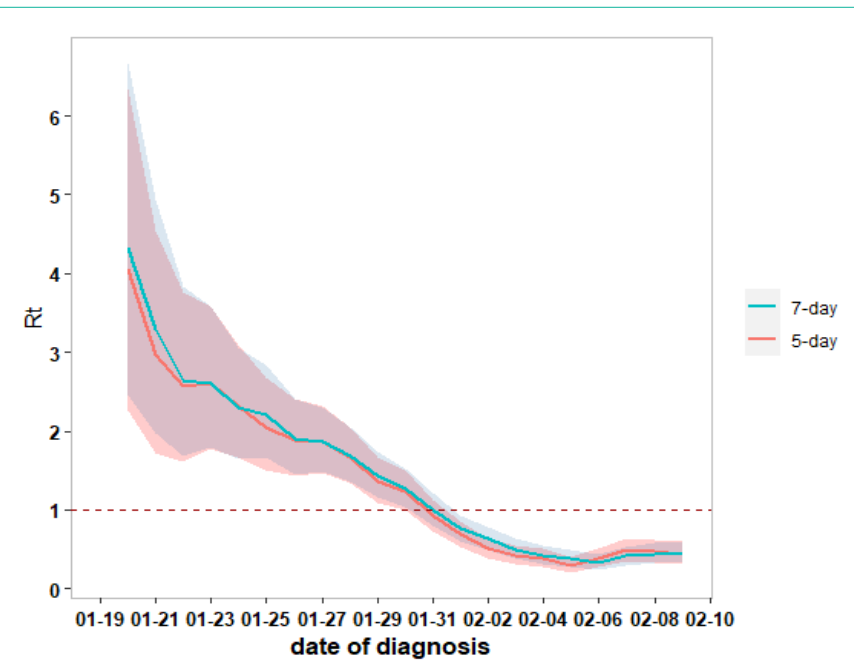

Figure 6: Real time reproduction number $\left(R_{t}\right)$ over a 5-day and 7-day moving average.

\section{Transmission route}

Based on complete epidemic history data of confirmed cases in Jiangsu Province, transmission routes of COVID-19 were classified into four types. Type one was for imported cases who had a clear contact history in Hubei, and then were confirmed a few days after isolation (Figure 5, patient 1,2), with or without local transmission. Type two was for clustering cases in a family, in which situation, family members were transmitted by other infected family members (Figure 5, patient 3,4). Type three was entertainment aggregation transmission, in which situation, patients were infected by person who carried the virus through close contact in congregation or party (Figure 5, patient 5,6). The last type was unconscious contact transmission (Figure 5, patient 7,8), in which situation, patients were infected by unconscious contact with confirmed COVID-19 patients, such as staying in the same train cabin with infector. The local second-generation infected patients under these modes may again generate three-generation or more-generation infected cases through the above transmission routes, forming a complete route of transmission.

\section{Estimation of reproduction numbers}

The real time reproduction number $\left(R_{t}\right)$ is the average number of secondary cases generated by a typical primary case at time $t$, and $\mathrm{R}_{0}$ is the initial reproduction number before onset peak. We estimated the $\mathrm{R}_{0}$ by maximum likelihood from Jan 14 to Jan 23 , considering the imported and local cases, and the mean (7.5) and standard deviation (3.4) of serial interval we used come from earlier research report on COVID-19 [2]. The $\mathrm{R}_{0}$ we estimated is 2.89 (95\% CI 1.57-4.80). The estimated $\mathrm{R}_{\mathrm{t}}$ over a 5-day moving average reached a peak value of 4.05 (95\% CI 2.27-6.34) on January 20, then began to decline quickly. The $R_{t}$ was below the epidemic threshold $\left(R_{t}<1\right)$ and maintained a continuing downward trend after January 31 . We also calculated the $\mathrm{R}_{\mathrm{t}}$ over a 7-day moving average in case the impact of the weekend on diagnostic test of COVID-19. The 7-day moving average had a peak $\mathrm{R}_{\mathrm{t}}$ of 4.32 (95\% CI 2.47-6.67), which showed a similar trend over time to the five-day moving average (Figure 6).

\section{Prevention and control measures}

In the early stage of the outbreak of Novel Coronary Pneumonia, the People's Government of Jiangsu Province successively initiated the first-level response to public health emergencies according to the (Emergency Response Plan for Public Health Emergencies in Jiangsu Province) on January 24. It also highlighted the focus of work to strictly preventing imported epidemics, including strictly managing the agricultural market, doing a good job in the ventilation, disinfection, temperature detection, and health quarantine of personnel-intensive places such as airports, stations, and docks, and public transportation. The hospital strengthened emergency protocols, and medical personnel improved their own safety protection to effectively ensure early detection, early reporting, early diagnosis, early isolation, and centralized treatment measures. It was forbidden to assemble, and it was necessary to close large entertainment venues, continue to track close contacts, etc., cut off the chain of communication in time, and require residents to reduce going out and wear masks in public places (Figure 7). At the same time, on January 27, the Jiangsu Health Committee issued a document, proposing 'strengthen epidemic surveillance and implement a reporting system. standardize medical treatment and ensure patient safety [10]. Manage in accordance with laws and regulations to prevent the spread of the epidemic; promptly disclose information and strengthen propaganda and guidance. Actively strive for support and strengthen logistics support.' and further eight specific requirements. All of above affirmed the timeliness of the previous epidemic prevention work, and made a more detailed system of arranging epidemic prevention work from the aspects of mission, monitoring, treatment, logistics, etc.at the same time.

\section{Discussion}

In terms of geographical distribution, until March 18, the cumulative number of confirmed patients in Nanjing, Xuzhou, and 


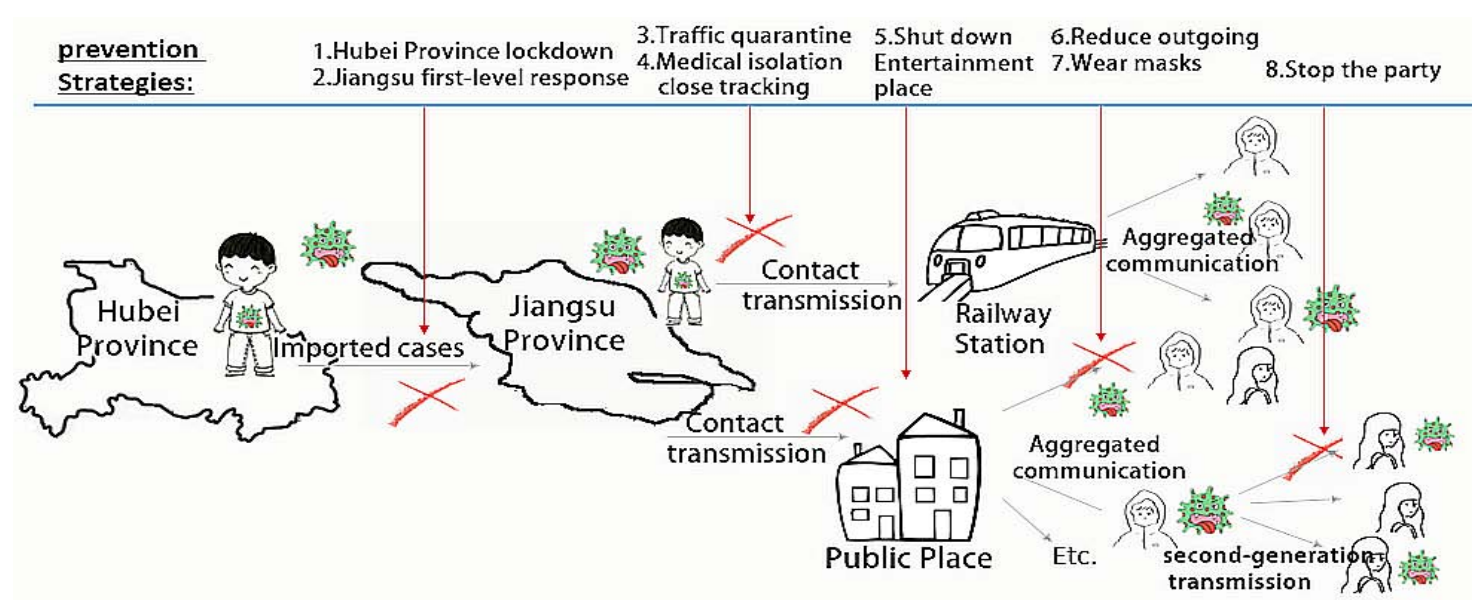

Figure 7: Schematic diagram of epidemic prevention measures in Jiangsu Province.

Suzhou ranked the top three in the province. The three cities also had the highest proportion of imported cases. According to the transportation network map of Jiangsu Province (Figure 2D), Nanjing and Xuzhou are the largest road and railway transportation hub in Jiangsu province, and Suzhou has extremely convenient railway and road traffic with Shanghai, the international transportation hub. The above data suggested that the convenience of transportation of the region was relative to the number of imported cases. A higher number of imported cases could increase the risk of local transmission in the lack of effective prevention and control measures. Before the twentieth century, it would take months for the pandemic to spread primarily due to limited national/international transport connectivity and slow trans-port systems. The emergence of modern transportation systems increases the transmission rate of the disease and the extent to which the pandemic may spread [9]. The epidemic prevention work at airports, stations, and railways would have the top priority.

In terms of time distribution, the epidemic curve of confirmed cases showed three stages: upward, plateau and downward. The first case was confirmed on January 22 and only one week later the highest number of the confirmed cases occurred. The peak lasted a week, then began to decrease. There were no new confirmed cases after February 18 (Figure 3). These findings were consistent with $R_{0}$ and $R_{t}$ results. Some cases cannot be confirmed in time due to insufficient detection capacity at the beginning of the epidemic, so the date of diagnosis of COVID-19 cases is 7 (5-10) days behind the date of onset.

In the early stage of the outbreak of COVID-19, $\mathrm{R}_{0}$ value of 2.89 (95\% CI 1.57-4.80) indicated that the transmissibility of COVID-19 is higher. This is related to the lax control measures and unawareness of COVID-19. $R_{t}$ values decreased rapidly as Jiangsu province and its cities started the first-level response to public health emergencies on January 24. It took one week that $R_{t}$ value dropped below the epidemic threshold after the control measures of "external against impo $R_{t}$ ed cases, internal against epidemic spread" were taken by the government (Figure 6). $\mathrm{R}_{t}$ refers to the average number of secondary cases generated by a typical primary case at time $t$ [10-12]. If $R_{t}$ is less than 1, it means that the epidemic of infectious diseases can be gradually controlled by maintaining the current prevention and control measures. If $\mathrm{R}_{\mathrm{t}}$ is greater than 1 , it means that infectious diseases will continue to spread, and the prevention and control measures need to be optimized and strengthened.

In addition, the Jiangsu Provincial Government and the Jiangsu Health Committee attached great importance to possible imported cases. On March $5^{\text {th }}$ and $26^{\text {th }}$, they issued a document to emphasize the procedures for handling the epidemic situation after travelers arriving in Nanjing. Work links such as screening of fevered passengers, determination of close contacts, retention of fevered passengers, etc., make clear requirements for the entire work flow of fever passengers for inspection and medical treatment [13]. At the same time, high attention was paid to the safety issues in the work of returning to work.

There were still some limitations in this study. Because the epidemic data were collected from the official websites of Jiangsu Commission of Health, the complete individual information, such as the specific onset time, exposure history, and past history of each case, clinical manifestations, diagnosis and treatment plans, treatment effects and personal basic conditions, etc. were unavailable. We failed to do a more in-depth study on the population distribution, susceptibility factors and so on. Besides the number of asymptomatic and presymptomatic cases of COVID-19 remained unclear and we only analyzed the epidemic characteristics of confirmed cases reported by the government websites. Furthermore, in the context of the international outbreak of novel pneumonia, until March 18, 2020, Jiangsu Province had reported none new case in the province for 27 consecutive days, and no new imported cases have been found. It can be considered that the epidemic in this province was close to disappearing. However, the epidemic prevention work still needed to be highly valued, guarded against the importation of foreign cases.

\section{Conclusion}

In conclusion, the analysis of the epidemiology and transmission dynamics of COVID-19 reported by Jiangsu Province as of March 18, 2020 , and the analysis of the prevention and control measures during the epidemic can lead to the following conclusions: 631 patients infected by COVID-19 were all discharged from hospital, with no death in Jiangsu province. It is crucial to interrupt local spread by taking strict control measures at the beginning of the epidemic. The 
war time prevention and control measures taken by the government were contributed to block the spread of COVID-19 in Jiangsu province. Although the epidemic situation has been basically under control up to February 18, more effective preventive measures are still needed for the resumption of work and production.

\section{Declarations}

\section{Ethics approval and consent to participate}

All data in this article comes from the official report of the Health Commission, and no privacy and ethics issues are involved.

\section{Availability of data and materials}

Supplementary data and codes were uploaded in Github.

The URL is as following: https://github.com/liujs1/Jiangsu_ COVID19.

\section{Funding}

This research was funded by Natural Science Foundation of China (81872718); Health Commission of Shanghai Municipality (201840041); 2018 medical education research project of medical education branch of Chinese Medical Association and medical education professional committee of Chinese Higher Education Society (2018b-n02081); Key undergraduate course project of Shanghai Education Commission (2019-65). The funders had no role in the design of the study and collection, analysis, and interpretation of data and in writing the manuscript.

\section{Authors' Contributions}

YC, JL and CY contributed to study design, introducing function fitting methods, mathematical calculation, data analysis and processing, figures plotting, literature search and paper writing. AZ, ZA contributed to $\mathrm{aR}_{\mathrm{t}}$ icle quality supervision and overall control.

\section{Acknowledgements}

Thanks to all medical workers on the frontline fighting against this epidemic.

\section{References}

1. Huang C, Wang Y, Li X. Clinical features of patients infected with 2019 novel coronavirus in Wuhan, China. Lancet. 2020; 395: 497-506.

2. Li Q, Guan X, Wu P, et al. Early transmission dynamics in Wuhan, China, of novel coronavirus-infected pneumonia. New England Journal of Medicine. 2020; 382: 1199-1207.

3. Chen N, Zhou M, Dong X, et al. Epidemiological and clinical characteristics of 99 cases of 2019 novel coronavirus pneumonia in Wuhan, China: a descriptive stud. Lancet. 2020; 21: 30211-30217.

4. Jiangsu Commission of Health. China Commission of Health.

5. China CDC. The epidemiological characteristics of an outbreak of 2019 novel coronavirus diseases (COVID-19) in China. Chinese Journal of Epidemiology. 2020; 41: 145-151.

6. National Novel Coronavirus Pneumonia Medical Treatment Experts Group. Novel Coronavirus Infected Pneumonia Diagnosis and Treatment Program. 2020.

7. National Novel Coronavirus Pneumonia Medical Treatment Experts Group. Novel Coronavirus Infected Pneumonia Diagnosis and Treatment Program. 2020-219.

8. National Novel Coronavirus Pneumonia Medical Treatment Experts Group. Novel Coronavirus Infected Pneumonia Diagnosis and Treatment Program. 2020.

9. Ali Y, Sharma A, Haque MM. Transportation and a Pandemic: A Case Study of COVID-19 Pandemic. In: Indian Institute of Technology, National Institute of Disaster Management (eds) Integrated Risk of Pandemic: COVID-19 Impacts, Resilience and Recommendations. Disaster Resilience and Green Growth. Springer, Singapore. 2020.

10. Thomas O, Romana H, Pierre-Yves B. The RO package: a toolbox to estimate reproduction numbers for epidemic outbreaks. BMC Medical Informatics and Decision Making. 2012; 12: 147-147.

11. Thompson RN, Stockwin JE, van Gaalen RD, et al. Improved inference of time-varying reproduction numbers during infectious disease outbreaks. Epidemics. 2019; 29: 100356

12. Zhang J, Litvinova M, Wang W, et al. Evolving epidemiology and transmission dynamics of coronavirus disease 2019 outside Hubei province, China: a descriptive and modelling study. Lancet Infect Dis. 2020; 20: 793-802.

13. China daily. Jiangsu Commission of Health System Actively Prevents and Controls Novel Coronary Pneumonia. 2020. 